Filol. linguist. port., n. 14(2), p.191-202, 2012.

\title{
Analyse textuelle des discours: niveaux ou plans $d^{\prime}$ analyse
}

Jean-Michel Adam

Résumé: L'article porte sur la théorie de l'Analyse Textuelle des Discours, à partir d'une reprise de la traduction brésilienne de La linguistique textuelle: introduction à l'analyse textuelle des discours (Cortez, 2008). L'ATD est pensée en fonction de trois observations préliminaires: la linguistique textuelle est une des disciplines de l'analyse de discours, le texte est l'objet d'analyse de l'ATD, et, dès qu'il y a texte, c'est-à-dire reconnaissance du fait qu'une suite d'énoncés forme un tout de communication, il y a effet de généricité, c'est-à-dire inscription de cette suite d'énoncés dans une classe de discours. Le modèle théorique de l'ATD est éclairé par une reprise de son schéma 4, où sont représentés huit niveaux d'analyse. L'ATD est abordée sous l'angle d'une double exigence - des raisons théoriques et des raisons méthodologiques et didactiques qui conduisent à ces niveaux - et sont détaillées et illustrées les cinq plans ou niveaux d'analyse textuelle. Pour finir, des parties de l'œuvre sont reprises et élargies, avec d'autres analyses où de nouveaux aspcts théoriques sont détaillés.

Mots-clés: Analyse textuelle des discours. Linguistique textuelle. Plans d'analyse. Niveaux d'analyse.

\section{CADRE THÉORIQUE DE L'ANALYSE TEXTUELLE DES DISCOURS}

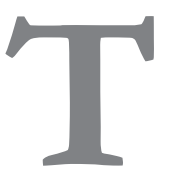

out le monde connait, au Brésil, P Analyse de discours. De même la Linguistique textuelle est reconnue dans ce pays grâce aux travaux importants d'Ingedore Grunfeld Villaça Koch et de Luiz Antonio Marcuschi. Mais ces deux domaines ont connu des développements parallèles avec peu de recoupements. La particularité de A lingüistica textual, Introdução à análise textual dos discursos (São Paulo, Cortez, 2008) est de réunir ces deux champs de recherche dans une théorie unifiée que j'ai appelée, depuis un certain nombre d'années Analyse Textuelle des Discours (port. Análise Textual dos Discursos - ATD). L'originalité de ce 
second Simposio d'ATD ${ }^{1}$ est d'illustrer et d'explorer ce domaine, dont les deux autres particularités résident, d'une part, dans l'intégration de l'étude des textes littéraires dans l'approche des faits de discours en général, et d'autre part, dans la mise en avant des aspects textuels et descriptifs de la traduction.

Trois remarques préalables vont me permettre de préciser le cadre de l'Análise textual dos discursos (ATD) :

- Remarque 1 : la linguistique textuelle est une des disciplines de l'analyse de discours. Définie comme un champ interdisciplinaire, l'AD a besoin d'une théorie de la langue en emploi (Saussure parlait de «langue discursive ») qui ne peut éluder la question du texte comme unité d'interaction humaine. La linguistique textuelle est donc un sous-domaine de l'AD.

- Remarque 2 : le texte est l'objet d'analyse de l'ATD. Il est la trace langagière d'une interaction sociale, la matérialisation sémiotique d'une action socio-historique de parole.

- Remarque 3: Dès qu'il y a texte, c'est-à-dire reconnaissance du fait qu'une suite d'énoncés forme un tout de communication, il y a effet de généricité, c'est-à-dire inscription de cette suite d'énoncés dans une classe de discours. En d'autres termes, il n'y a pas de textes sans genre(s) et c'est par le système de genre d'une formation socio-historique donnée que la textualité rejoint la discursivité et que la linguistique textuelle retrouve l'analyse de discours.

Parler d'ATD implique une double exigence que je schématise dans le schéma 4 de la page 61 d'Adam (2008), dont je propose ici une révision partielle :

1 J-M .Adam refere-se ao II ${ }^{\circ}$ Simpósio Internacional de Análise Textual dos Discursos (Natal, UFRN, novembro 2010) 


\section{Esquema 1}

Análise textual dos discursos

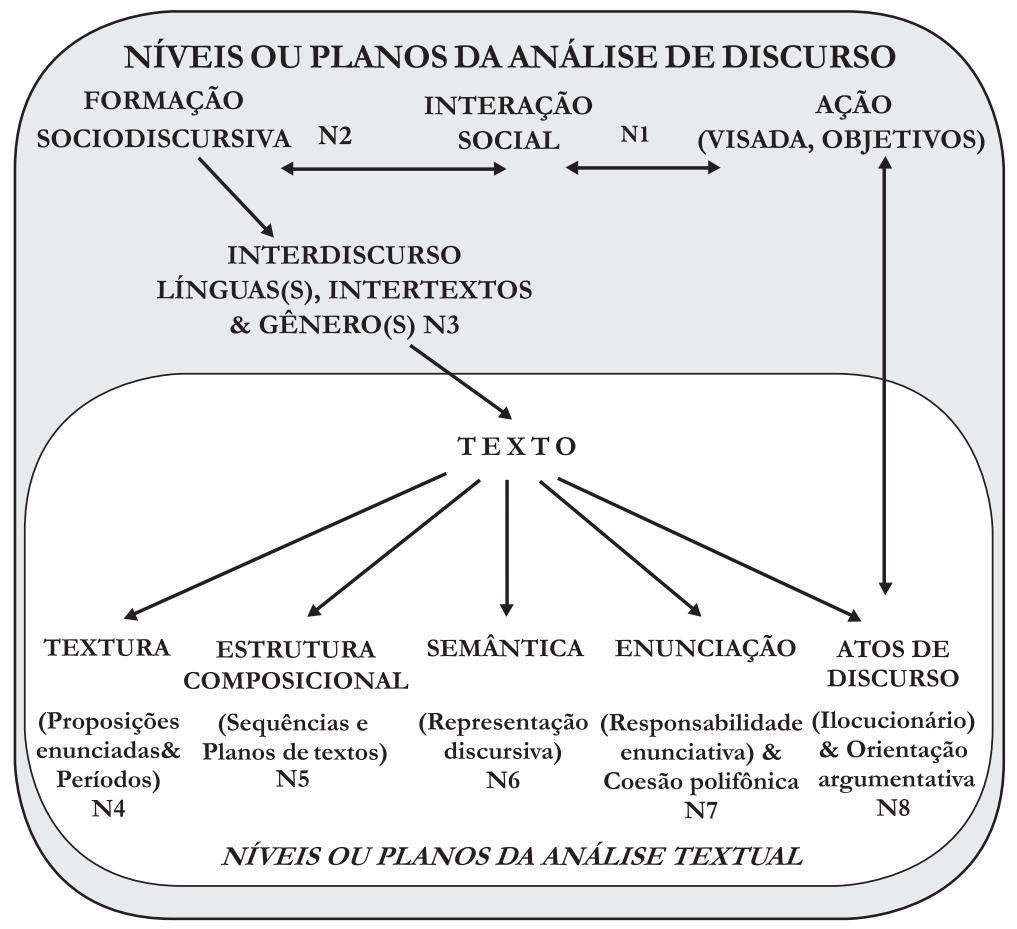

La particularité de mon modèle théorique tient à la distinction des 8 niveaux d'analyse que détaille le schéma 1 . Des raisons théoriques et des raisons méthodologiques-didactiques m'ont amené à retenir ces différents niveaux ou plans d'analyse.

- Des raisons théoriques : il existe des théories partielles pertinentes de ces différents niveaux. Ainsi la théorie des actes de discours ou actes illocutoires (Austin, Searle, etc.) est une théorie partielle du niveau N8; la théorie des genres est une théorie du niveau N3 ; la théorie des séquences textuelles que j'ai développée est une théorie partielle du niveau N5 ; la linguistique de l'énonciation (Benveniste) et les théories du point de vue (Rabatel, Nølke) sont des théories du niveau N7 ; la théorie de l'argumentation dans la langue (Ducrot) est une théorie des niveaux N8 et N6. Le niveau N1 est parfaitement théorisé par les chercheurs qui se positionnent dans l'interactionnisme social (Bronckart) et c'est l'objet principal 
des théories interactionnistes et conversationnelles, tandis que le niveau N2 est l'objet classique de l'analyse de discours française (Pêcheux), bien connue au Brésil.

- Des raisons méthodologiques et didactiques : la complexité de l'objet d'étude est telle, qu'il est méthodologiquement nécessaire de le diviser et de distinguer des moments d'analyse et même de théorisation. Chaque niveau constitue à mes yeux un moment d'analyse, une unité de recherche et d'enseignement (c'est un aspect didactique que je considère comme très important) liée aux autres mais assez distincte des autres pour former un tout. En fait, on peut décrire un texte en se contentant d'un niveau d'analyse et en s'appuyant sur une théorie consistante de ce niveau. Le tout est de bien voir qu'on n'opère alors qu'une description partielle d'un objet de très haute complexité qui demande une théorie plus vaste.

Faute de place, je ne dirai qu'un mot du niveau N3 que je considère comme un niveau d'analyse essentiel. C'est d'ailleurs ce que confirment séparément l'analyse de discours et la linguistique textuelle. L'analyse de discours a fait de la question des genres une question fondamentale, comme les travaux de Dominique Maingueneau en sont un bon exemple. Dans le domaine de la linguistique textuelle, sous l'influence des théories anglo-saxonnes (depuis Gülich, 1986), une distinction classique traverse la littérature théorique brésilienne : Textypen (Tipos de textos) et Textsorten (Gêneros de textos), ces derniers étant totalement séparés du concept littéraire de Gattung (Genres littéraires). En sortant la question des genres de la linguistique textuelle proprement dite (schéma 1), je propose de parler de gêneros de discursos en réunissant Gattung et Textsorten sous une même enseigne (N3) et je considère les Tipos de textos (Textypen) comme des dérivés des "tipos de seqüências" de niveau N5 (Adam, in M. Cavalcante, 2009: 115-132). Les genres sont des catégories de la production (auctoriale), de l'édition (éditoriale) et de la réception (lectoriales). Ces trois instances de généricité peuvent se recouper ou diverger ; les systèmes de genres évoluant dans le temps, ce phénomène est sensible pour tous les textes qui ont une durée d'existence prolongée (littéraires, religieux, philosophiques, par exemple). 


\section{LES CINQ PLANS OU NIVEAUX DE L'ANALYSE TEXTUELLE}

Pour illustrer les niveaux d'analyse N4 à N8, je vais compléter le schéma 10 de la page 111 en le réarticulant sur la partie basse du schéma 1.

\section{Esquema 2}

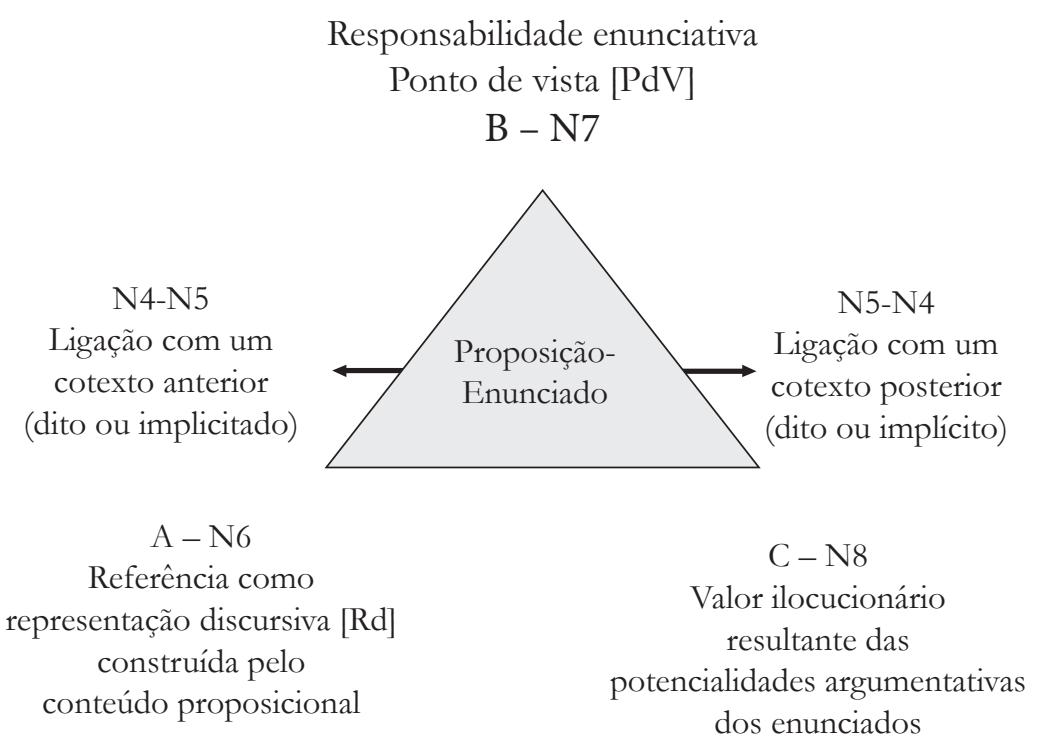

Je parle de "proposition $»^{2}$ - sans préciser chaque fois micro-proposition afin de souligner le fait qu'il s'agit à la fois d'une micro-unité syntaxique et d'une micro-unité de sens. Je retiens de la proposition classique le lien entre un objet du discours (« sujet» ou « thème ») et ce qui en est dit à l'aide (énoncé verbal) ou non (énoncé nominal) d'un prédicat verbal. Toute proposition-énoncé comporte trois dimensions complémentaires auxquelles s'ajoute le fait qu’il n'existe pas d'énoncé isolé : même apparaissant seul, un énoncé élémentaire répond à un ou plusieurs autres et/ou en appelle un ou plusieurs autres en réponse ou simple continuation. Cette condition de liage (niveaux N4 et N5) est en grande partie déterminée par l'orientation argumentative de l'énoncé. Les trois dimensions complémentaires de toute proposition énoncée sont : une dimension énonciative [B-N7] qui prend en charge la représen-

2 Ce qui suit est partiellement déjà traduit, pages 108-112, de Adam (2008). 
tation construite verbalement d'un contenu référentiel [A-N6] et lui donne une certaine potentialité argumentative qui lui confère une force ou valeur illocutoire plus ou moins identifiable [C-N8].

La modalisation énonciative, travaillée en profondeur par l'argumentation, explique l'extrême diversité de réalisation de cette valeur illocutoire des énoncés. Par exemple, /Féliciter/ peut aussi bien être rendu par un énoncé monorème ayant valeur de simple acte expressif : «Bravo !», ou par un énoncé performatif plus formel ayant soit la forme d'un énoncé verbal : «Je vous félicite », soit d'un énoncé nominal : «Toutes mes félicitations!» (accompagnant une poignée de main ou un mouvement de tête). À cela s'ajoute le fait énonciatif qu'un «Bravo !» modulé par un certain ton et/ou une expression du visage peut devenir un énoncé ironique et un blâme au lieu d'un compliment (le décalage entre l'énoncé et le contexte - une énorme maladresse ou gaffe par exemple rendant cette interprétation possible). La prise en charge énonciative ou point de vue $[\mathrm{PdV}]$ permet de rendre compte du dédoublement polyphonique propre à l'ironie : le locuteur $[\mathrm{L}]$ se dissocie $[\neq]$ d'un PdV1 (félicitation) pris en charge par un énonciateur E1 mis en scène dans et par sa propre parole, tandis qu'il s'associe [=] au PdV2 (reproche) d'un énonciateur E2. On peut ainsi schématiser la structure énonciative de tension polyphonique inhérente à l'ironie :

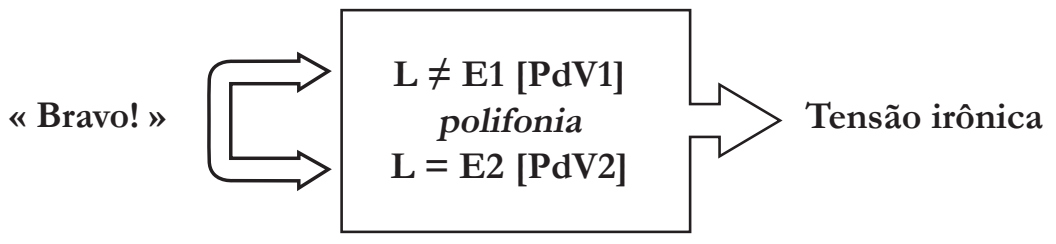

Le fait de placer la valeur descriptive de tout énoncé dans la partie [A-N6] n'implique pas son découplage des autres composantes. Il s'agit seulement de poser, à ce premier niveau, un certain nombre de faits. Après avoir défini tout acte de référence comme une construction opérée dans et par le discours d'un locuteur et comme une (re)construction par un interprétant, nous parlerons de représentation discursive $(\mathrm{Rd})$.

Nous localiserons en [A-N6] la question de l'évaluation de la valeur de vérité des énoncés selon deux régimes pragmatiques : celui de la vériconditionnalité qui repose sur l'opposition du vrai et du faux/mensonger et celui de la fictionalité, qui apparaît comme un régime du ni vrai ni faux dans lequel on peut intégrer la métaphoricité. Ces deux régimes sont largement déterminés par le genre de 
discours et/ou par les figures du discours (métaphore, symbole et allégorie dominant dans le second, distinguées de la métonymie, de la synecdoque et de la métalepse dominantes dans le premier). Au niveau énonciatif [B-N7], la combinaison d'une $\mathrm{Rd}$ et d'un $\mathrm{PdV}$ se superpose à l'alternative vériconditionnalité/fictionalité. À l'articulation des niveaux [A-N6] et [B-N7] se pose, en effet, la question de la validité plus que de la vérité des énoncés : un énoncé (une $\mathrm{Rd}$ ) est posé comme valide selon le locuteur (JE-valide), selon son ou ses interlocuteurs (VOUS-valide), selon les autres (délocutifs IL(S)/ELLE(S)-valide), pour tous (NOUS-valide) ou selon l'opinion commune, la doxa des maximes, proverbes et dictons (ON-valide). Le poids de validité d'un énoncé, qui relie donc une Rd [A-N6] et un PdV [B-N7], est un aspect essentiel de sa valeur argumentative et de sa valeur illocutoire [C-N8]. Ce que résume le schéma 2, par un triangle qui ne hiérarchise pas les trois composantes, qui situe $[\mathrm{A}]$ et $[\mathrm{C}]$ sur la même ligne (ce qui correspond au codage classique de la pragmatique des actes de discours) et place l'énonciation [B] en position médiane, entre $[\mathrm{A}]$ et $[\mathrm{C}]$.

À la lumière d'un exemple étudié pages 123-124 de Adam (2008) (le titre-chapeau d'une publicidade da rede ferroviária federal suíça sur la ville de Zurich), il est facile d'exemplifier les liens étroits entre les cinq composantes de base de toute proposition énoncée :

\section{(1) ZURICH}

\section{Cosmopolite}

et pourtant typiquement suisse

\section{ZURIQUE}

\section{Cosmopolita}

e, no entanto,

tipicamente suíça

Au niveau N4, ce fragment textuel est analysable dans son dispositif typographique et syntaxique particulier. La tête nominale est marquée par le nom propre en lettres capitales (ZURIQUE). Au lieu de faire suivre cette tête nominale par une prédication verbale, ce texte se présente comme une structure nominale Nom propre (ZURICH) + Adjectif (Cosmopolite), disposée sur deux lignes. Donner ainsi une propriété à un objet est une forme de prédication. ${ }^{3}$ 
Certes, cette disposition peu conventionnelle se prolonge, après le connecteur et pourtant (ligne 3), par l'énoncé d'une nouvelle propriété (ligne 4). Tout en opérant une séparation graphique, les alinéas relient les unités selon une même structure nominale : Nom propre + Adjectif $1+$ connecteur + [ellipse de la répétition du Nom propre] Adverbe et Adjectif 2. On a ici affaire à une structure très proche de celle des phrases nominales dont Benveniste (1966: 151-167) étudie le fonctionnement en indo-européen et en grec ancien . Selon lui, il existe deux modes distincts d'énonciation, qui forment l'un et l'autre un énoncé assertif fini. Les énoncés nominaux et verbaux sont, dans cette hypothèse, deux types concurrents et complémentaires d'assertion : « La phrase nominale ne saurait être considérée comme privée de verbe. Elle est aussi complète que n’importe quel énoncé verbal» (op. cit., p. 159). Tant que l'énoncé nominal était considéré comme une phrase verbale à verbe déficient, sa nature spécifique ne pouvait ressortir. En particulier le fait que « le prédicat de la phrase nominale, même lorsqu'il est adjectif, a [...] une valeur essentielle et exprime [...] une part intégrante de l'être du sujet » (id., 167). C'est bien le cas ici où il semble qu'un verbe être soit implicité : Zurique est cosmopolite (proposition p) Zurique est typiquement suisse (proposition q).

Benveniste (1966: 160) dit encore : «Une assertion nominale, complète en soi, pose l'énoncé hors de toute localisation temporelle ou modale et hors de la subjectivité du locuteur». Cette forme de sous-énonciation est importante à relever car le fait qu'il semble que « personne ne parle ici» (pour reprendre une formule chère à Benveniste) est une donnée importante de la construction du sens. Notre exemple exploite pleinement cette valeur sémantique qui dépasse le cas particulier des langues anciennes et de l'Irlandais du Kerry étudiés par Benveniste :

Étant apte à des assertions absolues, la phrase nominale a valeur d'argument, de preuve, de référence. On l'introduit dans le discours pour agir et convaincre, non pour informer. C'est, hors du temps, des personnes et de la circonstance, une vérité proférée comme telle. C'est pourquoi la phrase nominale convient si bien à ces énonciations où elle tend d'ailleurs à se confiner, sentences ou proverbes. (Benveniste, 1966: 165). 
Retenons que la notion de phrase graphique, définie par une complétude Sujet + Verbe encadrée par une majuscule et un point final, rend mal compte des multiples propriétés et possibilités non seulement de l'oral, certes, mais également de l'écrit. Les propriétés hétérogènes de la ponctuation consistent à marquer aussi bien l'intonation et l'énonciation que l'unité logico-grammaticale, le rythme et la mise en relief d'un segment textuel. Parmi les propriétés typographiques, il ne faut pas oublier la forme, la taille, la couleur des lettres et la place de l'énoncé dans l'espace graphique. Christophe Luc et Jacques Virbel parlent fort justement à ce propos de propriétés «typo-dispositionnelles » de la mise en forme matérielle des énoncés (2001). ${ }^{4}$

Le fonctionnement textuel doit, par ailleurs, être pris en compte. Au niveau N5, nous pouvons dire que cette structure périodique binaire : Proposition $p+$ Connecteur + Proposition q est une représentation discursive (A-N6) complexe qui prend la forme d'une période descriptive binaire ou séquence descriptive minimale. D'après la définition que j'en donne dans Adam (2008: 215-224), on est ici en présence d'opérations descriptives successives :

a.Operação de Pré-tematização ou ancoragem (denominação imediata do objeto que abre - escopo à direita - um período descritivo) : «Zurique » (pré-Th).

b.Operações de Aspectualização : qualificações ou atribuições de propriedades au pré-Thème : 1 «Cosmopolita » (Rd1-proposition p) et 2. « tipicamente suíça » (Rd2-proposition q).

Au niveau A-N6 s'ajoute une double connexion entre ces deux aspectualisations qui construisent deux représentations discursives $(\mathrm{Rd})$ concurrentes et posées comme contradictoires de la ville de Zurich. Cette contradiction correspond à une double vision du Thème-Zurich, à deux points de vue sur la ville : Rd1-proposição $\mathrm{p}=\mathrm{PdV} 1+\mathrm{Rd} 2$-proposição $\mathrm{q}=\mathrm{PdV} 2$. Le connecteur pourtant signale que ces deux PdV sont contradictoires.

Au niveau C-N8 des actes de discours, on a affaire ici à deux actes d'assertions successifs. Le passage de cette valeur descriptive-assertive pri-

A partir de là, je dis des choses nouvelles par rapport à Adam (2008). 
mitive à un macro-acte de discours à dériver de cette description se fait par l'interprétation de cette double assertion comme un acte de recommandation d'aller visiter la ville de Zurich au moyen des chemins de fer fédéraux. Cette dérivation illocutoire s'explique par l'orientation argumentative de l'énoncé. En effet, le connecteur concessif pourtant découpe l'énoncé global en deux propositions nominales élémentaires ( $p$ et pourtant $q$ ) argumentativement orientées dans un sens différent. Plus précisément $\mathrm{p}$ est un argument qui incite à tirer une conclusion ayant un sens opposé à $\mathrm{q}[=$ non-q]. Cette orientation argumentative de chaque proposition est inséparable de leur prise en charge énonciative. Pour qu'un même segment textuel puisse comporter deux sens contradictoires, il faut que les deux propositions soient prises en charge par des énonciateurs différents, qu'elles opposent deux PdV (PdV1 vs PdV2).

Le locuteur se présente comme reconnaissant une première logique qui oppose le cosmopolitisme à l'identité suisse (PdV1), mais il ne s'y arrête pas pour proposer une autre représentation qui, tout en comportant une norme négative implicite, conserve à la ville de Zurich sa propriété « typiquement suisse » (norme positivée, PdV2).

\section{Esquema 3}

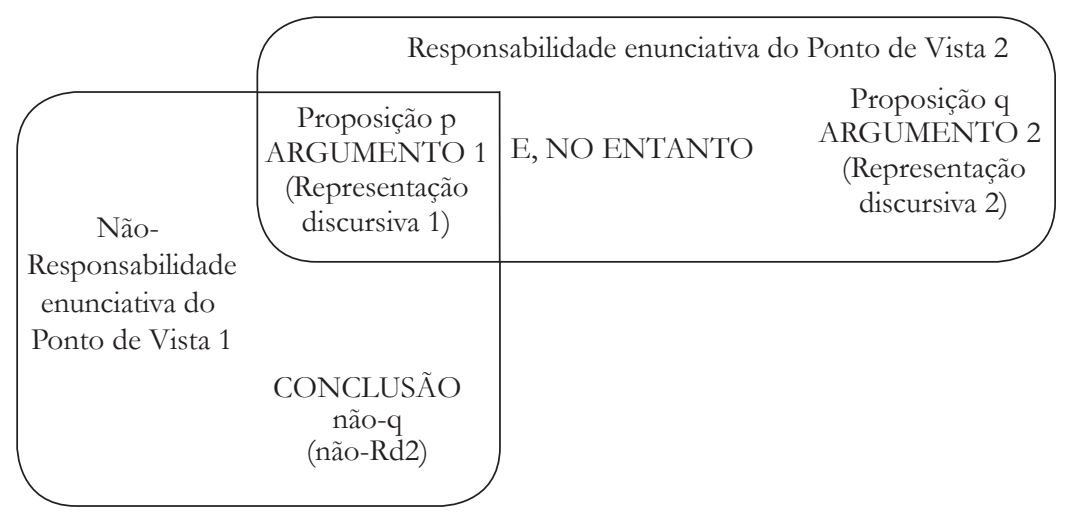

Tout cela s'éclaire quand on considère la suite du document. Le début et la fin du rédactionnel développent ce que le titre-chapeau synthétise (je souligne en gras les reprises, en italiques les éléments paraphrastiques et en petites capitales les indicateurs de présupposition): 


\section{(2) ZURICH}

\section{Cosmopolite}

et pourtant

typiquement suisse

Malgré son rayonnement international, Zurich

n'a pas perdu les qualités et le charme d'une

ville typiquement suisse. [...]

En flânant dans les ruelles pavées de la vieille

ville vous vous apercevrez bien vite que Zurich

n'a rien perdu du charme d'une petite ville ;

cosmopolite à souhait, elle reste « made in Switzerland».

La série ouverte par malgré (autre connecteur concessif) : « n’a pas per$\mathrm{du} »$, « n'a rien perdu », « reste », laisse entendre qu'en raison de son « rayonnement international » et de son caractère « cosmopolite», la ville aurait dû perdre ses caractéristiques nationales : « qualités et le charme d'une ville typiquement suisse/petite ville ». La logique sous-jacente de ce mouvement argumentatif apparait quand on constate dans le corpus du même document publicitaire da rede ferroviária federal suíça le retour du lexème « charme », répété deux fois à propos de Zurich et toujours rattaché au « made in Switzerland » et au retour également de la propriété « cosmopolite»:

\section{(2) GENÈVE}

\section{Ville cosmopolite}

au charme français.

\section{GENEBRA}

Cidade cosmopolita

Com charme francês.

Pour Genève, la même propriété « cosmopolite » est couplée, sans concession ni opposition, à un « charme » qui n'a rien d'helvétique. Si Zurich est encore une ville suisse, Genève ne l'est visiblement plus, selon le PdV suisse alémanique que cette représentation discursive laisse entrevoir. La comparaison de (1) et de (3) incite à associer la propriété « cosmopolite » à la perte potentielle de l'identité nationale. Dans le système du discours publicitaire des Chemins de Fer Fédéraux suisses, le mot prend un sens qu'il ne possède que de façon potentielle dans la langue. On reconnaît sous ces deux exemples la présence insidieuse d'un cliché de l'interdiscours nationaliste qui, depuis 
Maurras et Barrès, oppose cosmopolite à national. C'est ce cliché nationaliste qui induit l'inférence $p-R d 1>>$ conclusion non- $q$.

On voit comment, du bas du schéma 1 (niveaux N4 à N8), on peut remonter ainsi au haut du haut du schéma (niveaux N1 à N3) et, en particulier, à l'interdiscours sur lequel se fonde le document publicitaire.

\section{RÉFÉRENCES BIBLIOGRAPHIQUES}

Adam, Jean-Michel. A lingüística textual: introdução à análise textual dos discursos. São Paulo: Cortez, 2008.

Benveniste, Emile. Problèmes de linguistique générale 1. [traduction portugaise]. Paris: Gallimard, 1966.

Cavalcante, Mônica M. et al. (Org.) Gêneros e sequências textuais. Recife: Edupe, 2009.

Gülich Elisabeth. Textsorten in der Kommunikationspraxis. In: Kallmeyer, W. (org.). Kommunikationstypologie: Handlungsmuster, Textsorten, Situationstypen. Düsseldorf: Schwann, 1986. 15-45.

Koch, Ingedore G. V. Introducão à lingüistica textual. São Paulo: Martins Fontes, 2006.

Luc, Christophe; Virbel, Jacques. Le modèle d'architexture textuelle: fondements et expérimentation, Verbum XXIII, 1, P.U. Nancy, 2001. 103-123.

Marcuschi, Luiz A. Lingüística de texto: o que é e como se faz?. Ed. esp. São Paulo: Parábola, 2009 [1983].

\section{Análise textual dos discursos: níveis ou planos de análise}

Resumo: O artigo aborda a teoria da Análise Textual dos Discursos, a partir de uma retomada de suas linhas gerais, conforme encontradas na tradução brasileira de A linguística textual: introdução à análise textual dos discursos (Cortez, 2008). A ATD é situada em função de três observações prévias: a linguística textual é uma das disciplinas da análise de discurso, o texto é o objeto da ATD e, desde que há texto, isto é, reconhecimento do fato de que uma série de enunciados forma um todo comunicativo, há efeito de genericidade. O modelo teórico da ATD é esclarecido a partir da apresentação de um esquema (esquema 4) da obra, em que figuram diversos níveis de análise. Abordada sob a ótica de uma dupla exigência - as razões teóricas e metodológico-didáticas que levam a esses níveis -, são detalhados e exemplificados os cinco planos ou níveis de análise textual. Na continuidade, são retomados trechos da obra e expandidos, com outras análises e maior detalhamento da teoria.

Palavras-chave: Análise textual dos dicursos. Linguística textual. Níveis de análise. Planos de análise. 